\title{
Dialogando con Joan Romero González sobre Paulo Freire: El valor transformador de la educación, la formación permanente $y$ a lo largo de la vida ${ }^{1}$
}

Dialogue with Joan Romero González about Paulo Freire: The transforming value of education, permanent training and throughout life

Laura Peris Reig

Universidad Complutense de Madrid lauperis@ucm.es ORCID ID: https: / orcid.org/0000-0001-6251-4216

Fig. 1. Joan Romero en la actualidad

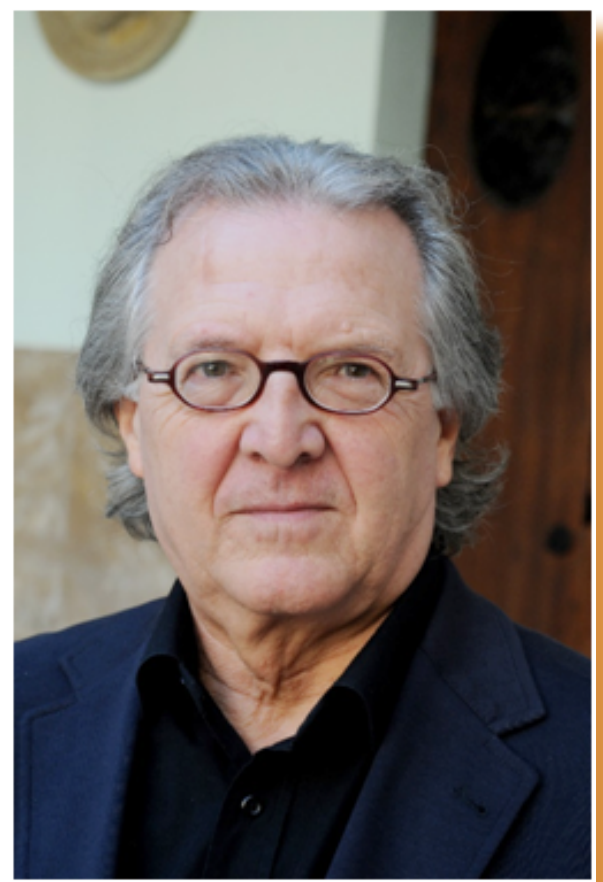

Joan Romero González es Catedrático de la Universidad de Valencia, donde actualmente desarrolla su trabajo docente en la Facultad de Geografía e Historia, concretamente en el Departamento de Geografía.

$1 \quad$ Mi agradecimiento al profesor Joan Romero González por darme la oportunidad de poder realizar esta entrevista facilitándome la libertad del cuestionario y regalándome generosamente su tiempo. 
Con un extenso y destacado currículum a sus espaldas, Romero ha sido Director General de Universidades de la Generalitat Valenciana [1983], Secretario General Técnico del Ministerio de Educación y Ciencia (1985-1987), Conseller de Educación y Ciencia y portavoz del Gobierno de la Generalitat Valenciana [1993-1995].También destaca su figura como director, hasta 2008, del Instituto Interuniversitario de Desarrollo Local [IIDL], además de impartir docencia en esta misma Universidad en diversas Titulaciones relacionadas con las Ciencias Políticas, el Periodismo, la Administración, y la Geografía e Historia. Además de profesor visitante en la School of Geography de Universidad de Leeds [Reino Unido] y galardonado con el Premio Extraordinario de Licenciatura y de Doctorado.

Numerosas publicaciones, conferencias, comunicaciones y ponencias de carácter nacional o internacional avalan su trayectoria personal y profesional, siendo autor/editor de 30 libros y casi un centenar de artículos y capítulos en libros. Entre sus publicaciones más recientes encontramos La secesión de los ricos [junto con Antonio Ariño, 2016] y la coordinación de Geografía del despilfarro en España [2019].

Durante estos últimos años, ha orientado su actividad docente e investigadora hacia el campo de las Políticas Públicas y la Geografía Política, habiendo sido miembro de la Comisión de expertos del Ministerio de Administraciones Públicas [2006-2007], así como del proceso de elaboración y discusión de la Estrategia Territorial de Navarra, Estrategia Territorial de la Comunidad Valenciana y Estrategia Territorial de Castilla-La Mancha.

Romero pertenece a la Cátedra Alfons Cucó de Reflexión Política Europea y a la Escola Europea de Pensament Lluís Vives en la Universitat de València. También, desde 2006 dirige las colecciones de publicaciones Desarrollo Territorial y Tirant lo Blanc de la Universitat de València, además de la Cátedra Prospect Comunitat Valenciana 2030, respaldada por la Presidencia de la Generalitat Valencia y la Universitat de València. Además de todo ello, el catedrático colabora de manera frecuente con diversos medios de comunicación.

De la figura del abogado y filósofo brasileño Paulo Freire, objeto de esta entrevista, el profesor Romero tuvo la oportunidad-gracias a Pep Aparicio, que les presentó-de conocerlo personalmente en el marco de una actividad formativa dirigida al profesorado, siendo Conseller de Educación y Ciencia de la Generalitat Valenciana, y lo recuerda porque son de esas cosas que le pasan a uno y no se olvidan, "no todos los días ves a Freire", reconoce.

Fig. 2. Paulo Freire y Joan Romero en el seminario organizado en Valencia, 30 de marzo de 1995. Fotografía de Carmen Montañana.

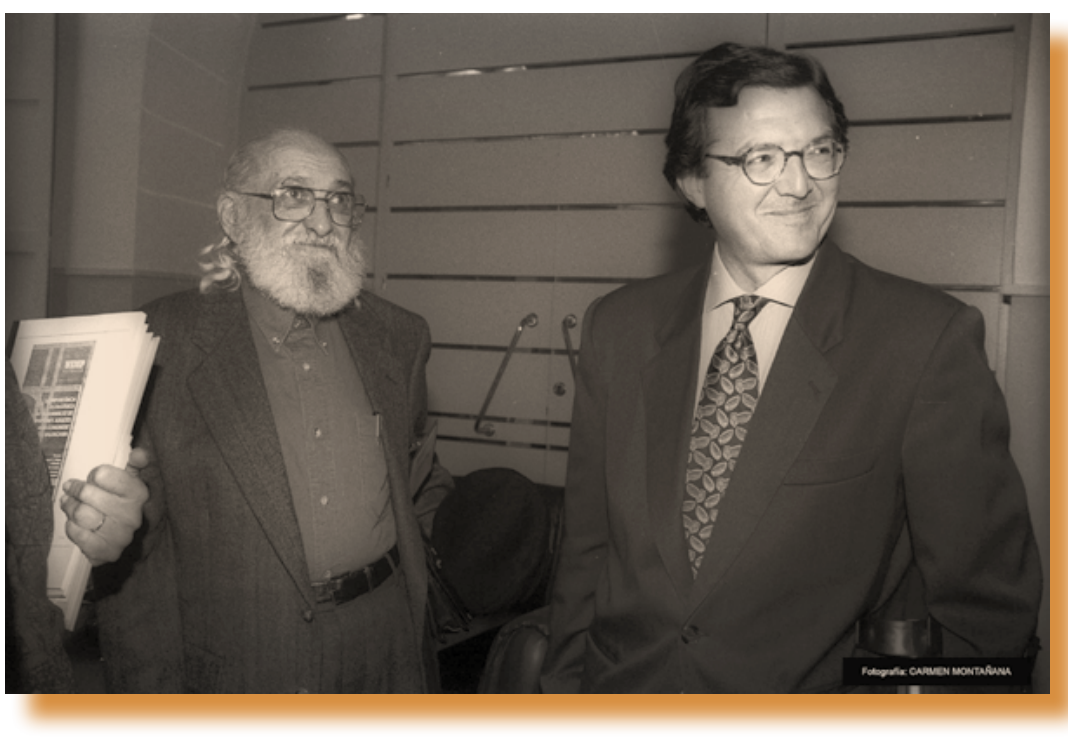


La entrevista con el catedrático Joan Romero ha ido más allá de la gran corriente del valor transformador de la educación y del posicionamiento crítico y activo que impregna toda la obra freireana. Comenzamos la sesión recordando la figura de Freire, destacando su filosofía más esencial y tratando de reconocer o identificar las influencias que podríamos encontrar a día de hoy en las políticas educativas más recientes. Así descubrimos que, para Romero, siempre habrá una parte muy importante del mundo de la educación heredera de lo que significaba la escuela o la gran corriente del valor transformador de la educación, entre los cuales uno de los pilares fue, sin duda, Paulo Freire. De esta manera, afirma: "sí que se podría decir que subsiste o pervive un hilo conductor en el que nos integramos una parte del mundo de la educación que comparte aquellos valores, aunque las coordenadas históricas y sociales en las que se consolida el pensamiento de Freire, naturalmente no tienen nada que ver con las que se han producido en los últimos 20 o 25 años". Romero se reconoce en principios de la obra de Freire y apunta que en este momento se ha llegado al final de un relato histórico que acabó prácticamente al finalizar el siglo XX, o incluso un poco antes, cuando las "sociedades sólidas" dieron paso a las "sociedades líquidas" propias del siglo XXI. En este sentido, el catedrático puntualiza:

"Considero que hemos llegado al final de un orden, o más bien al final de un relato histórico del que formábamos parte. Pero esto acabó, de modo que aquel mundo de certezas entrecomilladas, de sociedades sólidas, aquel orden que nace con la segunda guerra mundial acabó incluso un poco antes de que acabase el siglo XX. Acabó cuando las sociedades sólidas dieron paso a las sociedades líquidas, sin relato, sin perspectiva. El mundo comprimido en el presente, cargado de memoria, pero incapaz de proyectarse en el porvenir, es el mundo en el que vivimos en las últimas dos décadas. Al menos así lo define Enzo Traverso en su libro Melancolía de Izquierda: Después de las utopías, pues para él estaríamos ante un mundo comprimido en el presente sin vinculación con un relato que mire hacia atrás y también sin perspectiva pronóstica de futuro, de ahí la expresión de presente comprimido, y yo estoy totalmente de acuerdo con él."

Con ello diferenciamos que ahora los movimientos no son estructurales, no son continuos, son de otra forma, son efímeros, incluso los movimientos sociales. Romero lo visualiza como una forma de herradura por su constante dinamismo (rápidas subidas y bajadas). Por tanto, sí que podríamos encontrar influencias de Freire en las políticas educativas e incluso sociales actuales, pero no de la misma manera que en épocas anteriores.

Por otro lado, una de las preocupaciones de Freire era que la educación estaba siendo una educación de la respuesta y no de la pregunta, cuando, para él, la pregunta era en realidad la generadora de la curiosidad y de la reflexión que promueve el espíritu crítico. De hecho, Freire consideraba que todo conocimiento comienza por la pregunta, pero no tan solo la pregunta por la pregunta, sino utilizando aquellas capaces de estimular la curiosidad y promover la capacidad de asombro [Freire y Faundez, 2013]. Esto plantea el siguiente interrogante: “¿Cree que a día de hoy todavía falta más pedagogía de la pregunta tanto en la formación de niños y niñas, como de adolescentes y personas adultas?"A lo cual responde:

"Esta es precisamente la razón por la que no me he jubilado hasta ahora, por la cual sigo siendo profesor universitario. Cuando entro en el aula todavía creo que es posible trabajar justamente en esa dirección, por eso considero que la función fundamental del profesor es sin lugar a dudas la de ayudar a que se formulen preguntas, ayudar al alumnado a entender de manera crítica este mundo cambiante, lleno de incertidumbres e inseguridades, y al mismo tiempo acompañarles en el sentido más noble del término, es decir, acompañarles en el camino." 
Romero reconoce que el trabajo del profesor es un trabajo muy difícil, pero se ve inspirado por figuras como Josep Fontana o el mismo Freire, entre otros maestros, que le enseñaron otras formas de observarla realidad y entenderla, mientras él era estudiante. Pues ser profesor, dice, "es mucho más que trabajar en la enseñanza, de la misma manera que ser estudiante no es estar matriculado en la universidad, es muchísimo más”, y percibe que una parte de esto se está perdiendo actualmente, quizá por los cambios culturales, normativos o tecnológicos a los que se ve sometida nuestra sociedad actual, o por una combinación de los tres. Parecería así que la docencia entendida como elemento fundamental en la formación crítica de ciudadanos se está difuminando, devaluando, degradando. Lo cual, reconoce, le provoca cierta tristeza.

¿Y cuál sería el reto, entonces, que la filosofía de Freire plantea a los jóvenes docentes que empiezan ahora? Pues bien, según Romero, el reto radica en mantener viva la misión freireana, en tratar de ir más allá, en intentar cerrar las brechas sociales que todavía nos quedan por cerrar, al menos en España. Y confía en que se avive esta misión:

"Y es que pese a que soy consciente de que la meritocracia ha entrado en crisis, de que ya no es el ascensor social que fue hace 3 décadas o que tuvimos los de mi generación, el único ascensor social que tuvimos los que procedíamos de familias humildes como es mi caso; pese a saber también que el $80 \%$ de la vida en cualquier parte del mundo, también aquí, depende del país y de la familia en la que uno nace, y por último, siendo consciente así mismo de que "la pobreza se hereda", como dice Branko Milanovic, creo que todavía tenemos alguna misión."

Precisamente ahora, en tiempos de pandemia, Romero enfatiza más esta cuestión, pues asegura que el mayor riesgo que existe no solamente es que se agudice la divergencia o la fractura social, sino que todavía es posible que salgamos de ella en lo que él visualiza como una forma de "K". Es decir, que aún sea más rápida la separación entre un grupo de la población y otro grupo, lo cual es verdaderamente preocupante, especialmente en nuestro país, comenta.

"En España si miramos cualquier indicador nos damos cuenta de que ocurre algo curioso y es que hay algún tipo de anomalía, que yo no he sabido entender aún por qué, que nos hace un poco diferentes a otros países, diferentes a muchas democracias liberales de nuestro entorno, fundamentalmente en la gran cantidad de porcentaje de abandono escolar prematuro y del fracaso escolar que existe. Desde luego esto es una anomalía que debería ocupar a todos los poderes públicos en suturar esta profundísima herida. Es que es muy espectacular. En datos del año 2019, no del siglo XIX, España es el 4ํㅜ país de los 37 países miembros de la OCDE con la tasa más alta del número de repetidores; no digo que tengamos el $3 \%$ que tiene Finlandia, pero es que tenemos casi el 29\%, somos uno de los países donde los alumnos provenientes de familias más desfavorecidas repiten más, hasta 4 veces más que los procedentes de familias acomodadas y en algunas regiones hasta 5 y 6 veces más, por cierto, la Comunidad Valenciana entre ellas. Y esto se recoge en el informe COTEC de hace unos meses. Por tanto, esta sería la misión,esto es lo que yo les diría a los jóvenes que quieren dedicarse a esta noble misión de profesor y es que intenten cerrar brechas y cerrar el boquete todo lo que puedan sabiendo como sé que la meritocracia, el ascensor social, se ha averiado en gran medida."

Sin embargo, Romero reconoce que gran parte de esta misión ya no corresponde a los profesores, sino a los poderes públicos, y enumera una serie de propuestas o mandatos dirigidos a los poderes públicos. El primero sería que los poderes públicos se tomen el mundo educativo y al profesorado más en serio. Pues España es el país que arroja los peores resultados en materia de 
éxito escolar y al mismo tiempo es el país de todas las democracias liberales que más reformas educativas ha hecho. Esta es la gran paradoja. Cada gobierno lleva al parlamento una nueva ley. Sin embargo, ninguna de las numerosas leyes orgánicas que se han ido aprobando ha sido evaluada. Este sería el segundo mandato: evaluar sinceramente las reformas educativas que se han hecho en España. Y, por último, como tercer mandato, sugiere hacer un gran acuerdo por la formación, la educación y el aprendizaje permanente - mencionando estos tres últimos términos de manera diferenciada [pues no son lo mismo] - con voluntad de que perviva varias legislaturas, que perviva dos décadas.

El catedrático considera que este es precisamente el éxito que tienen otras democracias liberales y nosotros no. Nosotros, cuando llega un gobierno nuevo, lo primero que hace es sacar una reforma que ponga el acento en algún elemento simbólico para polarizar, a falta de un elemento central que haría que el ascensor social funcionara y que la doble red no se consolidara, porque han sido décadas de gobierno conservador que han trabajado con éxito en la conformación de dobles redes. Para Romero, si se llegase a hacer estos grandes acuerdos, si se cumpliesen los tres mandatos, tal vez nos iría mucho mejor.

Llegados a este punto, cabe rememorar algunos aspectos significativos del pensamiento freireano, en especial algunas de sus reflexiones acerca de la formación permanente y de los contextos teóricos en el marco de los escenarios y contextos formativos. Recordemos pues, como Freire considera que, en el contexto teórico de la formación permanente, se hace verdaderamente necesaria la reflexión crítica sobre los condicionamientos que el contexto cultural ejerce sobre cada individuo. Así, considera que la formación ha de inducir al análisis, a la investigación y a la reflexión crítica sobre cómo el contexto influye de muchas y diferentes maneras en las personas. Por ello, Freire cree que la persona debería estar en constante formación, es decir entiende la educación como un aprendizaje permanente a lo largo de la vida, y no solo como un acto o periodo puntual de la vida de un individuo.

Esta misma distinción se concreta en el Informe a la Unesco de la Comisión Internacional sobre la educación para el siglo XXI presidida por Jacques Delors (1996) y recogida en el libro La educación encierra un tesoro al diferenciar entre lo que sería la "educación básica" y la "educación permanente", de la siguiente manera:

"Para adaptarse realmente a las necesidades de las sociedades modernas, la educación permanente no puede ya definirse por referencia a un periodo particular de la vida - por ejemplo, la educación de adultos, por contraposición a la educación de los jóvenes - o una finalidad demasiado circunscrita, cuando se distingue, por ejemplo, la formación profesional de la formación general. En lo sucesivo, el periodo de aprendizaje cubre toda la vida, y cada tipo de conocimiento invade el ámbito de los demás y los enriquece. En vísperas del siglo XXI, la educación, debido a la misión que se le ha asignado y a las múltiples formas que puede adoptar, abarca, desde la infancia hasta el final de la vida, todos los medios que permiten a una persona adquirir un conocimiento dinámico del mundo, de los demás y de sí misma." [Delors, 1996, p.112]

Así, la educación básica, una vez logrados sus objetivos, puede suscitar el deseo de seguir aprendiendo, el cual puede tomar cuerpo en la continuación de estudios dentro del sistema oficial, o bien puede darse como un estudio ulterior.

Por su parte, la "educación durante toda la vida" se presenta en este documento como una de las 
llaves de acceso al siglo XXI, definiendo este término como un proceso continuo de educación que abarca toda la existencia y que se ajusta cada vez más a las dimensiones y ritmos de la sociedad y del mundo del trabajo, dando a cada sujeto la capacidad de conducir su destino en un mundo que cambia rápidamente y que se caracteriza por la creciente mundialización. Por ello, la educación a lo largo de la vida debe ofrecer a cada uno los medios necesarios para lograr un equilibrio entre el trabajo y el aprendizaje, obteniendo así una ciudadanía activa.

Y es que, para Freire, existe una estrecha relación entre ciudadanía y educación, pues considera que las problemáticas que atañen a la educación no se deben únicamente a aspectos relacionados con la metodología o la pedagogía, sino también con la política.

Por otra parte, cabe nombrar que también se ha hecho una distinción entre los conceptos de "educación permanente" - que parece asociarse más al derecho universal regulado y organizado por las políticas públicas a nivel de sistema - y "aprendizaje a lo largo de la vida" - que parece estar más relacionado con la responsabilidad individual y la libertad para que cada sujeto organice su propio proceso de aprendizaje en función de sus necesidades o intereses particulares-. Esta distinción permite que al hablar de educación permanente nos refiramos al proceso formativo que se da en pro de lograr una ciudadanía y una sociedad igualitaria y justa, mientras que al hablar de aprendizaje a lo largo de la vida se realzan más las cualidades personales, la adaptabilidad a las nuevas sociedades cambiantes y "líquidas", y a la competitividad entre unos sujetos y otros por poseer distintos tipos de formación [Besalú, 2012, p.25].

No obstante, ambas no serían incompatibles, sino complementarias, de manera que un sistema educativo puede estar orientado a formar ciudadanos preparados, críticos y justos dentro de una temporalidad concreta de su vida, y estos a su vez encargarse de gestionar su propio aprendizaje de manera permanente para ir adaptándose a cada situación de esta sociedad cambiante.

Romero, con respecto a si cree que actualmente nuestro sistema educativo está encaminado hacía un aprendizaje o una formación permanente, responde:

"Este es el cuarto punto débil de nuestro sistema y es que nosotros no disponemos de un sistema que incentive, que favorezca, que promueva el aprendizaje permanente en un momento en el que además será crítico por una doble razón, por eso lo de la salida en forma de "K" que se ha mencionado anteriormente. En primer lugar, porque nosotros somos un país que tiene una economía de bajos salarios, es decir, una economía que está centrada en una economía de servicios que requiere población o trabajadores que no necesitan mucha formación. Aparentemente creamos muchos empleos, pero a la primera de cambio destruimos mucho empleo también. La burbuja especulativa hizo mucho daño porque sacó todavía más a más gente del sistema educativo y la llevo a la construcción, a la economía de servicios. A esto hay que darle la vuelta por completo. Y, en segundo lugar, porque justamente ese es el modelo que irá quedando atrás y transitaremos a un modelo más basado en la productividad, en valor añadido a los procesos de producción, en personas que tengan un mayor nivel de formación y una mayor capacidad de aprendizaje, personas que sepan adaptarse a las destrezas digitales. Todo esto no lo tenemos. Además, se añade el hecho de que una vez acabada la etapa formativa dejamos a los ciudadanos a su suerte. Solamente alrededor de un $5 \%$ se estima que son las personas que estando en el sistema productivo retornan a los sistemas de formación."

Recurriendo a una metáfora, el catedrático compara nuestro sistema educativo con una forma 
de reloj de arena. ¿Qué quiere decir esto? Pues que está delgado en la parte central [que es la parte de la enseñanza media superior y de la formación profesional superior], es muy grueso en la parte baja [que es la formada por la educación básica, la educación obligatoria y por las personas todavía sin formación], y está bastante alta en la parte de arriba [que corresponde a los estudiantes con titulación superior]. Sin embargo, si vemos el sistema educativo de otros países vecinos, como Alemania, tienen forma de diamante; la diferencia es muy significativa, pues esta forma de diamante representa que la parte más gruesa es la central (a diferencia de la nuestra, que es la más estrecha] donde una parte muy importante de jóvenes y de adultos están más vinculados con sistemas de formación profesional, de reciclaje de entradas y salidas.

En este tipo de sistema también los gobiernos locales tienen más protagonismo, así mismo, la formación de personas adultas es más flexible, más vinculada a gobiernos locales, lo cual considera que no se ha dado en nuestros centros, a pesar de que la ley de Formación de las Personas Adultas, promulgada en el año 1995 siendo él Conseller de Educación y Ciencia abogaba por ello. Esta ley, vigente hoy en día, apostaba ya entonces por una coordinación completa entre educación y trabajo, junto con la construcción de una red de centros de FPA singulares, pero que no se ha desarrollado en su totalidad. De ella dice también sentirse orgulloso porque ejemplifica la importancia que da a la cuestión de los acuerdos y considera exitosa por ser: "muy trabajada desde abajo, desde los grupos, desde los colectivos" - elemento decisivo en la línea freireana -, y manifiesta especial interés en mostrar su reconocimiento al trabajo participativo del profesor Pepe Veiga, así como el obtenido por parte de las plataformas y colectivos de agentes sociales lo que considera fue enormemente importante para el éxito del texto legislativo elaborado y aprobado en el parlamento valenciano sin ningún voto en contra.

Así mismo considera que en nuestro sistema educativo todo esto está por hacer, y además es muy complicado conseguir logros si continuamente cambiamos las leyes (hasta por los mismos partidos], por lo que será difícil que salgamos de una economía de bajos salarios y, como consecuencia, la juventud y el futuro de varias generaciones de jóvenes lo tendrán muy difícil porque seremos un país de servicios, y "porque un modelo productivos de bajos salarios es esto, y con el agravante de pertenecer a una sociedad cambiante en la que todo va muy de prisa, como la que caracteriza el presente siglo, podemos perder bastantes vagones".

Podemos concluir, por tanto, que a largo de esta entrevista Joan Romero nos ha hecho conocer cómo son las sociedades líquidas que caracterizan el siglo XXI, muy diferentes a las llamadas sociedades sólidas - como a la que Freire perteneció -. También ha dejado claro que debemos salir de una economía caracterizada por los bajos salarios y que los poderes públicos tienen muchas responsabilidades en estas materias. Así como que la misión de Freire sigue aún hoy viva en nuestra sociedad y en nuestras escuelas, aunque bajo una perspectiva distinta a la de épocas pasadas.

Dejemos a los lectores algunas cuestiones para su reflexión: ¿Se está haciendo suficiente por parte de los responsables públicos para que nuestra sociedad no pierda vagones en materia de formación y educación?¿Cómo de conscientes somos de la necesidad de contar con una formación permanente y un aprendizaje a lo largo de la vida? Y, en definitiva, ¿Qué podemos hacer nosotros para rescatar la misión de Freire y abogar por una pedagogía crítica como la que él mismo promovía? 


\section{Referencias}

Besalú, X. [2012]. El debate cultura: la lucha por el significado. En M. Martí y J. Gil, Aprendizaje permanente: competencias para una formación crítica: aprender a lo largo de la vida (pp. 25-37). Edicions del Crec. Disponible en: http://institutpaulofreire.org/ downs/coe26.pdf

Delors, J. y otros [1996).La Educación encierra un tesoro. Informe a la UNESCO de la Comisión Internacional sobre la educación para el Siglo XXI. Madrid: SantillanaUNESCO Disponible en: http://innovacioneducativa.uaem.mx:8080/innovacioneducativa/ web/Documentos/educacion tesoro.pdf

Freire, P. y Faundez, A. [2013]. Por una pedagogía de la pregunta. Crítica a una educación basada en respuestas a preguntas inexistentes. Editorial Siglo Veintiuno. Disponible en: https://aprendizajesparalelos.files.wordpress.com/2016/08/paulo-freire-y-antoniofaudez-por-una-pedagogia-de-la-pregunta.pdf 